\title{
Natural Interaction for Card Games on Multiple Devices
}

Andreas Dippon

Technische Universität

München

Boltzmannstr. 3

85748 Garching

dippona@in.tum.de

Gudrun Klinker

Technische Universität

München

Boltzmannstr. 3

85748 Garching

klinker@in.tum.de

Permission to make digital or hard copies of part or all of this work for personal or classroom use is granted without fee provided that copies are not made or distributed for profit or commercial advantage and that copies bear this notice and the full citation on the first page. Copyrights for third-party components of this work must be honored. For all other uses, contact the owner/author(s). Copyright is held by the author/owner(s) ITS'13, October 6-9, 2013, St. Andrews, United Kingdom.

ACM 978-1-4503-2271-3/13/10.

http://dx.doi.org/10.1145/2512349.2514916

\begin{abstract}
In this poster we present the development process of natural interaction for card games on multiple devices. Our goal was to provide users with an application that can be interacted with similar to real cards. It is a critical part of actual game play that all users can observe clearly what actions are performed by each player. To imitate such flexible use of cards in real games, we did not implement any game rules in our system. Rather, we strived towards making all computer-related user interactions as clear and visible to all players as manipulations of real cards. For the development of our system we used an iterative user-centered design approach.
\end{abstract}

\section{Author Keywords}

Multi-display environments, multi-display interaction, natural interaction, user-centered design

\section{ACM Classification Keywords}

H.5.2. [User Interfaces]: User-centered design

\section{Introduction}

With the rapid spread of touch devices came many different ways of interacting with these. Due to this fast development, some gestures are nowadays considered common knowledge. Yet many of these gestures were developed in respect to the scenario of a single person 


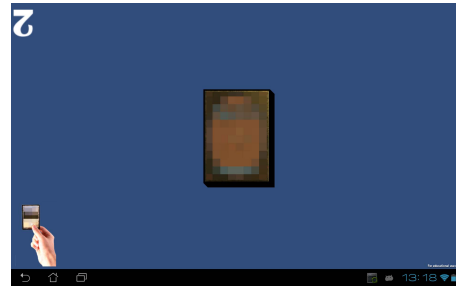

Figure 1: Table Application ${ }^{1}$

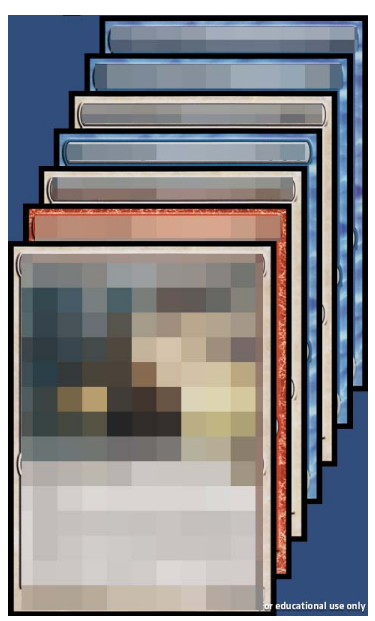

Figure 2: Hand Application interacting with the system. With the increased interest in the field of multi-display environments the question of how interaction works in such an environment arises. Additionally, developers also need to focus on the mental model of a user. This problem even multiplies, if other people need to understand the interactions performed by the user of such a system.

In order to tackle these questions, we focus on a specific scenario: card games. In card games, each player needs to continuously observe the actions taken by other players. How can this necessity to clearly understand the actions of other players be maintained, if these are using inter-process communications between multiple computers/displays? How do you define gestures for a virtual card game using multiple devices? How do users perceive the game experience?

\section{Related Work}

Multi-display environments in general have already seen a lot of research, e.g.[5]. There is also some more specific research on transferring data between displays available, that uses pick and drop gestures[6].

To find fitting gestures for interactions in a multi-display environment, prior work suggests that gestures benefit from being user defined[4]. Instead of inventing new gestures for virtual card games, observing players performing gestures in real card games and mapping these to the computer system seemed promising.

There is also some research on virtual card games on tabletop displays available[2]. Lobunets et al.[3] provided the players with a table application that showed a pile of

${ }^{1}$ Pictures of cards are blurred throughout the paper due to Intellectual Property Rights. cards to interact with. The user interactions were performed via buttons, e.g. users were able to transfer cards between their mobile phones and the table application.

\section{Game Description}

We chose the trading card game Magic the Gathering[1] as a test scenario. A standard game of Magic is played by two players competing against each other, each bringing their own pile of 60 cards. Our system simulates the game area and pile of one player as well as the cards in the player's hand. The player can then use the system to play against an opponent who is using real cards. As the game can become very complex, the players need to focus on the game and therefore should not be distracted by the user interface or the gestures of the system. The game also provides a lot of different interactions that the player needs to be able to perform, e.g. searching the pile for a card or placing cards behind other ones on the game field. Another aspect is each player's awareness of what the opponent is doing at all times. Therefore it is important that the player using the system can not perform any actions that do not fit in the mental model of playing the card game and that all actions can be followed and understood by the opponent as well as if he was using real cards.

\section{System Design}

The two parts of the system are the Table Application (see fig.1) and the Hand Application (see fig.2). The Table Application simulates the playing area showing public information. It is running on a tablet device. The Hand Application is used for showing the cards in a user's hand (private information). It is running on a smartphone. 


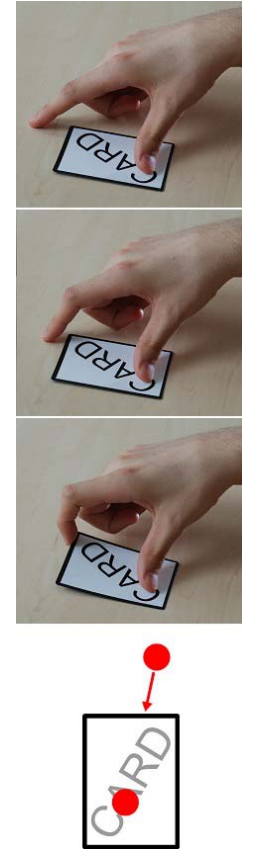

Figure 3: Pick Up One Card

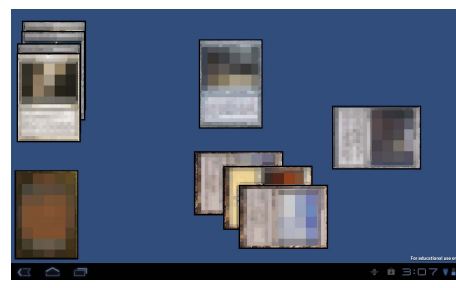

Figure 4: Version 1 Board
Table Application

The table application shows all public information. In addition to the cards, the virtual system needs to show the number of cards in a player's hand as it can not be seen on the backside of the smartphone. Another information that needs to be shown is a visualization of the transitional state when a user has picked up a card and is in the process of moving it to the cards in his hand. In principle, the card could go anywhere at this point in time. It is important that all players can be aware of a card in transitional state. To this end, we introduce the concept of the active hand, which is holding and moving a card between the public and the private display. The concept of the active hand is derived from the interaction with real cards. For example when a card is picked up from the table, it does not directly appear between the other cards in the passive hand. The player has to pick it up with one hand and bring it to the cards in the other hand. Because a virtual card can not be seen in the player's active hand a virtual hand icon appears on the table screen.

\section{Hand Application}

The hand application shows the cards that are in a player's hand. The cards can be sorted and picked up face up or face down. When the number of cards exceeds a certain threshold, the visualization changes, as the size of the cards would get too small.

\section{Gestures}

In multitouch systems, gestures for picking up or moving cards are typically abstracted to tapping and dragging gestures. These differ significantly from real grabbing and manipulating gestures that players apply to real cards. It is our hypothesis that players will be able to focus more on the game, if multitouch systems accept gestures that are as close as possible to the natural gestures of card manipulation - even though players may already have been exposed and familiarized with standard tapping gestures.

Therefore we developed such gestures by observing players during real card games. The implemented gestures for the table application include: Pick Up One Card, Pick Up Several Cards, Drawing a Card from the Pile, Lift Cards, Place Cards. For the hand application: Pick Card, Pick Card (face down), Put all Cards from Hand onto Table (face down), Shuffle Pile. The principle of the gesture design is shown with the help of the Pick Up One Card (see fig.3) gesture: A card is usually picked up by placing one finger on the card and moving another finger from outside the card towards the edge of the card. This interaction is directly mapped in the system. If one touch point is on a card and another touch point that started outside of the card is hitting the card, the card gets picked up into the virtual hand.

\section{Iterative Design Process}

Each of the four versions was tested with 2-3 expert users, who play the game regularly. The experts had different experience with multitouch devices, ranging from no experience to daily use. All experts were male and aged between 20 and 35 .

The first version (see fig.4) needed to be a very simple prototype to get feedback of players as unbiased as possible. It implemented all the basic functions needed to be able to play a game. Cards could be drawn from the pile by a simple tap. When a card from the hand got played, it appeared directly on the table application.

Cards could be picked up from the table by dragging them to the bottom of the screen. Players reported, that they would prefer more natural gestures when interacting with the cards. This could also improve the awareness of other players on what interactions are performed. Another very 


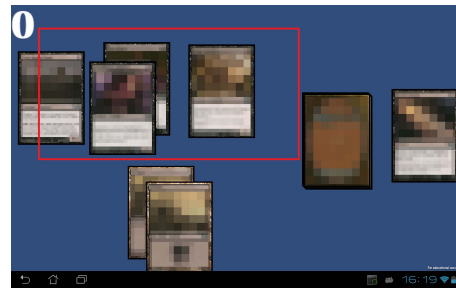

Figure 5: Version 2 Board

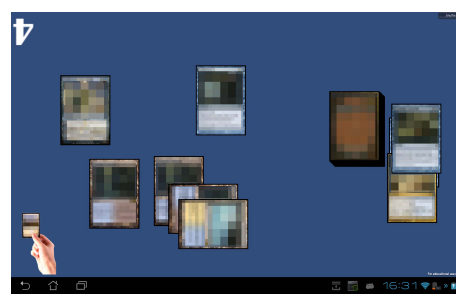

Figure 6: Version 3 Board

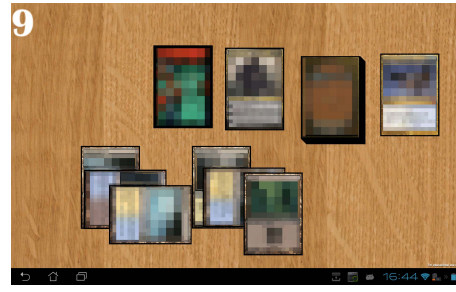

Figure 7: Version 4 Board interesting finding was that the players mentally treated the border of the tablet as a border of the game area. In a few games, the player with the system had to put some game tokens onto the game area, which was not supported. The players solved this problem by using rea cards and placed them next to the tablet. As the players needed to concentrate on the game and the real cards were outside of the tablet, having a different size than the virtual cards, the tokens were sometimes forgotten.

We added most of the player proposed features in the 2nd version (see fig.5). We also integrated a layer between the hand and the table when a card is moved from the hand to the table. In order to put a card from the hand to the table the player had to place it actively on the tablet after removing it from the hand. Another new interaction mode was to draw a selection rectangle around several cards and subsequently interact with all selected cards at once. The selected cards were highlighted on the screen. The evaluations showed that the players were able to play much more fluently with the new version. The players were quite fond of the new interaction of playing a card from the hand, as it resembles the movement made when playing with real cards. They also reported that the use of the selection rectangle is very strange, as it feels completely unnatural.

After receiving the same feedback about natural gestures from different experts in both versions, we implemented the gestures described in the Gestures section above. According to the feedback, we also removed the selection rectangle from the program but added the option to interact with several cards at once that are behind each other. Similar to real cards, a touch is propagated through the top card it touches to other cards below that card. This version (see fig.6) was received very well during the evaluation. The participants needed a few minutes to get used to the natural gestures. Afterwards they could play fluently with the system and the opponents were well aware of the interactions the player performed. Yet the propagation of the touches to cards behind each other caused a lot of problems when players wanted to drag a single card out of a few stacked cards.

Figure 7 shows the current version of the system. We removed the touch propagation from the previous version and added a different visualization for the hand application if the number of cards in the hand exceeds a certain threshold. Overall we changed some aspects of the visual appearance of the game to make it more appealing.

\section{References}

[1] Magic the Gathering. http://www.wizards.com/magic.

[2] Döring, T., Shirazi, A. S., and Schmidt, A. Exploring Gesture-Based Interaction Techniques in Multi-Display Environments with Mobile Phones and a Multi-Touch Table. In AVI2010 (May 2010).

[3] Lobunets, O., and Prinz, W. Evaluating a Smart Working Environment with a Digital Card Game Prototype. In CSCW'11 (Mar. 2011).

[4] Morris, M. R., Wobbrock, J. O., and Wilson, A. D. Understanding Users' Preferences for Surface Gestures. In Gl '10 (June 2010).

[5] Seyed, T., Burns, C., Costa Sousa, M., and Maurer, F. From Small Screens to Big Displays: Understanding Interaction in Multi-Display Environments. In IUI '13 Companion (Mar. 2013)

[6] Wilson, A. D., and Benko, H. Combining Multiple Depth Cameras and Projectors for Interactions On, Above and Between Surfaces. In UIST '10 (Oct. 2010). 